\title{
Jubilados en Twitter. Análisis de narrativas y seminalidad en los tuits de personas mayores para la discusión de temas públicos. El caso de Chile
}

\author{
María de los Angeles Miranda Bustamante \\ Universidad de Playa Ancha, Valparaíso, Chile
}

\begin{abstract}
RESUMEN
El presente estudio se pregunta acerca de las conductas digitales de las personas mayores en Twitter, una plataforma donde se comentan profusamente las noticias y los asuntos públicos, considerando la importancia de que estos usuarios se conviertan en usuarios activos de internet, y desde allí participen en la construcción de la realidad social y a la vez puedan encaminarse hacia un envejecimiento activo. La investigación, a través de una metodología cuantitativa, analiza las narrativas textuales y digitales en una muestra aleatoria de tuits de usuarios chilenos de más de 60 años. Además se calcula la seminalidad de estas publicaciones, es decir, la capacidad de producir interacciones con otros usuarios. Los resultados sugieren un alto interés por comentar asuntos públicos en la plataforma, una conducta tendiente a la convergencia mediática y el despliegue de esfuerzos digitales para interactuar con otros tuiteros. Sin embargo, aún existe una tasa de error técnico del 10\% y una bajísima seminalidad de las publicaciones, lo que indica la necesidad de mejorar la alfabetización digital en este segmento etario.
\end{abstract}

Palabras Clave: Twitter, personas mayores, seminalidad, narrativas.

\section{Retired people on Twitter. Analysis of narratives and seminality of elders' tweets commenting on public affairs. The case of Chile}

\section{ABSTRACT}

The purpose of this research was to examine the digital behaviors of the elders on Twitter, a platform where news and public affairs are profusely commented. The topic is important, considering that, becoming active digital users, older people could participate in the social construction of reality and move towards the so-called active ageing. The study, using a quantitative methodology, analyzed textual and digital narrative in a random sample of tweets, written by Chilean users over 60 years old. In addition, seminality was calculated in these posts, in order to evaluate the ability to produce interactions with other tweeters. The results suggest a high interest in commenting on public matters, a media convergence trend and the use of digital tools in the attempt of interacting with others. Nevertheless, there still exists a $10 \%$ rate of technical errors and an almost null seminality of the posts that indicate the urgent need to improve digital literacy in this age segment.

Keywords: Twitter, elderly, seminality, narratives.

El envío de cartas al director para opinar sobre los aciertos o males de nuestra sociedad fue durante muchos años una de las pocas formas de interactividad del público con los antiguos medios de comunicación, que permitía incidir en algo en sus contenidos (Richardson, 2008). Sobre todo era aprovechado por adultos mayores, quienes, tras una intensa vida laboral, disponían de algo de tiempo para reflexionar y escribir sobre el entorno. Al menos en Chile, las tribunas del lector aún existen, aunque cada vez menos lectores las alimentan y siguen. En esto ha influido sin duda la migración de los usuarios a soportes digitales, donde no sólo leen informaciones sobre el ámbito público, sino también pueden comentarlas, compartirlas o rebatirlas instantáneamen- te. De ahí que no sea difícil pensar en la posibilidad de que, al menos una pequeña parte de los escritores mayores de cartas al diario, estén intentando trasladar sus comentarios a una red social como Twitter.

Por esta razón, el presente estudio parte de la necesidad de abordar la relación entre los adultos mayores y las tecnologías digitales tomando a las personas de más de 60 años como sujetos-usuarios activos, productores de contenidos, considerando su progresiva integración digital, dejando de lado la idea de que todos los miembros de la tercera edad son espectadores-otros, marginados por la brecha digital etaria. Hasta ahora los estudios sobre este tema se centran mayormente en el necesario diagnós- 
tico de la situación de los adultos mayores que no participan en internet y las redes sociales y en los problemas que los llevan a esta exclusión. En contraste, el objetivo de esta investigación es describir el uso de Twitter por parte de las personas mayores en Chile, que utilizan estas redes para comentar las noticias, tal como solían hacerlo a través de las cartas al director, un área donde apenas se esbozan algunos pocos datos científicos.

De este modo, a través de una metodología mixta, se pretende analizar las narrativas textuales y digitales que despliegan los tuiteros mayores de 60 años que generan discursos digitales con publicidad para complementar, contrastar y argumentar en torno a las temáticas de contingencia pública, participando de este modo en la construcción social de la noticia, lo que además contribuye a su bienestar individual y a su envejecimiento activo. Se plantea de este modo la hipótesis de que en Chile existe un grupo pequeño de adultos mayores que son tuiteros activos y manejan las estrategias digitales básicas para invitar a otros usuarios a la interacción, comentando temas de interés social.

Este objetivo cobra notoriedad tomando en cuenta que el reciente censo realizado en Chile estableció que los mayores de 65 años se han casi duplicado en los últimos 25 años (INE, 2017). Además, las proyecciones auguran que en 2020 llegarán a ser un $17 \%$ de los chilenos (INE, 2012).

Pese a ello, la tendencia a analizar la relación entre los adultos mayores e internet desde un punto de vista deficitario y no desde el usuario activo, puede deberse a estereotipos negativos muy arraigados en Chile. De hecho, el estudio de Nazar y Figueroa (2015) comprueba que estos esquemas cognitivos preconcebidos se dan en el país tanto entre quienes conviven a diario con personas mayores como entre quienes no lo hacen, e incluyen valoraciones negativas, como la lentitud para actividades mentales y la dificultad en el manejo de la tecnología.

\section{Estado del arte}

Los estudios que abordan la relación de los adultos mayores con las redes sociales digitales, coinciden en la importancia y utilidad de estas aplicaciones para reactivar sus círculos sociales (Ciboh, 2017, Sæbø, Resca, \& Spagnoletti, 2015) y sus relaciones con amistades. Al mismo tiempo, les sirven para mantenerse informados de la vida de sus familiares y amigos (Girlado, Leal, \& Lilia, 2015) y también del acontecer social, con el potencial de llevarlos hacia el envejecimiento activo (Alves \& De Oliveira, 2015).

Pese a lo anterior, la gran mayoría describe una brecha digital definida por la edad (Niehaves \& Plattfaut, 2014). De este modo, los estudios encontrados se enfocan principalmente en los procesos de adopción de tecnología, usando para el análisis el Technology Adoption Model (TAM) (Ramón-Jerónimo, Peral-Peral, \& Arenas-Gaitán, 2013) o el Technology Adoption Propensity Index (TAP-I) (Farias, Vitor, Lins, Pedroza, \& Eduardo, 2015). De este modo, buscan evaluar cuáles son las barreras de acceso de los adultos mayores a los sitios web de redes sociales, especialmente por una falta de adaptación en el diseño de las interfaces (Chou, Lai, \& Liu, 2013). Asimismo, se establece que los adultos mayores presentan dificultades en el manejo digital en comparación con grupos etarios más jóvenes (Tsai, Chang, \& Ho, 2016) y se abordan, entre otras aprensiones considerables, las sensaciones de dependencia, vulnerabilidad y discapacidad en el uso de las tecnologías (Farias et al., 2015).

De todos modos, existen investigaciones que concluyen que las habilidades digitales de las personas de más edad no pueden caracterizarse de forma homogénea (Dos Santos Tarallo \& Gasparetto Sé, 2016) y que éstas dependen en gran medida de la posibilidad de contar con formación especial (Farias et al., 2015), de la asistencia de alguien más joven (Sæbø et al., 2015) o de las características específicas del grupo familiar (Rasi \& Kilpeläiner, 2015). Otra mirada alternativa considera esta brecha etaria como un estereotipo y que más bien existen barreras psicológicas, dado que un adulto mayor podría tener una edad cognitiva más joven, que le permita estar más abierto a las innovaciones digitales (Peral-Peral, Arenas-Gaitán, y Villarejo-Ramos, 2015).

Se pudo establecer que sólo un pequeño grupo de investigadores ha descrito el rol activo de los adultos mayores en las redes sociales digitales, pero principalmente a nivel de usos y gratificaciones (Jung, Walden, Johnson, \& Sundar, 2017), intentando descubrir sus motivaciones principales y sus percepciones de utilidad versus riesgos. Entre los beneficios más valorados, se incluyen la comunicación con sus cercanos, la entretención y el ocio y, muy particularmente, la autoformación y la búsqueda de información y de noticias, que en España alcanzó el nivel más alto de las preferencias entre los adultos mayores, según el estudio de Agudo, Pascual, y Fombona (2012). En contraste, se cuenta entre sus recelos la sensación de pérdida de la privacidad. De hecho, el estudio de Lung et al. (2017) concluye que los adultos mayores que usan Facebook procuran atender las solicitudes de sus contactos, pero sin un exceso de "social disclosure", es decir, revelando muy poco de sí mismos. De hecho, a pesar de las supuestas barreras tecnológicas, existe una tendencia de los adultos mayores, al menos en Facebook, a cambiar las preferencias de privacidad que vienen por defecto y a revelar fotos y datos de sí mismos sólo si otros de sus contactos también lo hacen (Chakraborty, Vishik, \& Rao, 2013).

Entonces, considerando la heterogeneidad de este grupo y las ya expuestas cifras crecientes de su uso de redes sociales, se puede afirmar que pocos esfuerzos investigativos se han concentrado en caracterizar a los adultos mayores como efectivos usuarios de las redes sociales. Sólo se exceptúa el estudio de Peral-Peral, Arenas-Gaitán, y Villarejo-Ramos (2015), que reconoce que existen adultos mayores que tienen una edad cognitiva menor a su edad cronológica, que les permite convertirse en verdaderos prosumidores y gestores de discursos relacionados con la vida privada y social.

En esta perspectiva, podemos escasamente añadir la investigación sobre la percepción del tiempo en los adultos mayores usuarios de las redes sociales en Rusia, que plantea que los recursos digitales, al carecer de sustancia física, cambian la memoria colectiva y la construcción del pasado, provocando efectos como la disolución y sincronización del tiempo (Sergeyeva \& Makarova, 2016). No se pudieron encontrar investigaciones sobre los discursos y narrativas de los mayores en redes sociales, sobre sus comentarios en línea acerca de las noticias ni tampoco un esbozo sobre los usuarios activos y productores de discursos pertenecientes a este grupo etario.

\section{Chilenos mayores probando internet}

Pese a los antecedentes ya descritos, en Chile, tomando la nomenclatura de Rogers (1983) sobre la difusión de las innovaciones, no podríamos encasillar a los adultos mayores en el área digital como los rezagados, sino como la mayoría tardía, la que se toma tiempo para contrastar los pros y contras de la innovación antes de adquirirla. Esto porque, pese a que en nuestro país este grupo tiene los ingresos mensuales más bajos (INE, 2015), entre 2013 y 2016 creció la cantidad de personas mayores que tienen un smartphone, de un 3,6\% a un 20,1\% (Fernández y Herrera, 2016).

El mismo estudio descubrió que el uso del teléfono celular para conectarse a las redes sociales también ha crecido en este 
segmento, pero especialmente en quienes tienen mejor nivel educacional. Esto sugiere que la formación es clave en la adopción de las innovaciones digitales, más que los ingresos y una supuesta inhabilidad relacionada con la edad. De hecho, las cifras indican que más de un tercio de los adultos mayores que asistieron a la universidad, usa las redes sociales a través del móvil.

También ha crecido la capacidad de hacer tareas digitales básicas sin ayuda, como escribir un correo electrónico. En 2013, sólo un $14,8 \%$ de los adultos mayores lo habrían hecho por sí mismos. Este indicador subió a un $22,6 \%$ tres años después. Lo que llama la atención es que el porcentaje se empina bruscamente a un $60,1 \%$ si se trata de usuarios que asistieron a la educación superior. Por lo tanto, nuevamente podemos concluir que, en el acceso digital de los mayores, la educación demuestra ser un factor clave (Fernández y Herrera, 2016).

Además, una investigación realizada en la Pontificia Universidad Católica de Chile establece que hay un acceso mayoritario de los mayores de 60 años a internet $(93,7 \%$ ) (Condeza et al., 2016). Además puntualiza que, aunque siguen prefiriendo la televisión, un $16,5 \%$ usa internet para conocer noticias, porcentaje que aumenta si se trata de temas de cultura $(23,3 \%)$ y salud $(44,6 \%)$. Y, según un antext: Why Older Adults Use or Avenior.e a 2020álisis de escenario proyectado en Chile a 2020, se espera que siga aumentando el uso de tecnologías digitales entre los usuarios senior (Godoy y Gálvez, 2015).

De esta manera, es plausible inferir la existencia de un grupo creciente de adultos mayores activos en internet que buscan noticias en línea y que utilizan las redes sociales para compartirlas y comentarlas.

\section{Envejecimiento activo}

El contenido y las narrativas de los discursos digitales de los adultos mayores son cruciales para motivar y orientar políticas de e-inclusión públicas y privadas. Sobre todo porque la participación en redes sociales digitales tiene una dimensión de bienestar individual para las personas mayores, en primer lugar, porque las protege del aislamiento social, tomando en cuenta que en Chile un $19,1 \%$ de los miembros de este segmento se siente aislado; un 20,1\%, excluido y un 35,3\% expresa que le falta compañía (Fernández \& Herrera, 2016).

Las redes sociales tienen un efecto en esa línea. El estudio de El-Sahili (2014) destaca la posibilidad en Facebook, por ejemplo, de desarrollar conductas "como si", que se producen simulando estar frente a una asamblea o audiencia o en lugares que no se han visitado nunca. Además, se reconoce que las redes sociales aumentan el capital social, usando el término de Bourdieu (1980), aun cuando se trate de vínculos débiles (Ellison, 2007; El-Sahili, 2014; Morgan, 2014; Young, 2011). De hecho, en una investigación aplicada a los adultos mayores españoles, se afirma que el desarrollo de sus habilidades de comunicación en internet genera la admiración entre sus pares (Llorente-Barroso, Iñarás-Abad, \& Sánchez-Valle, 2015). Además, en Chile, se ha comprobado que la participación en organizaciones sociales aumenta las probabilidades de experimentar la sensación de bienestar (Fernández \& Herrera, 2016; Palma-Candia, Hueso-Montoro, Ortega-Valdivieso, Montoya-Juárez \& Cruz-Quintana, 2016; Rojas, Carvajal, \& Kornfeld, 2011), lo que también puede trasladarse a la participación discursiva virtual.

Estos beneficios concuerdan con el concepto de envejecimiento activo, patrocinado por la OMS, el que no sólo incluye el bienestar individual, sino la integración social efectiva:"The word "active" refers to continuing participation in social, economic, cultural, spiritual and civic affairs, not just the ability to be physically active or to participate in the labour force. Older people who retire from work, ill or live with disabilities can remain active contributors to their families, peers, communities and nations. Active ageing aims to extend healthy life expectancy and quality of life for all people as they age" (WHO, 2002).

Esto confirma la necesidad primero de hacer un diagnóstico profundo del uso de los adultos mayores de las redes sociales. No sólo de su interés y capacidad tecnológica para usarlas como ayuda práctica para su vida privada cotidiana, sino también de cómo se sumergen en ellas para insertarse en la sociedad.

\section{Narradores mayores}

Este estudio, al centrarse en la perspectiva de los adultos mayores como productores de contenidos digitales en Twitter, se enfoca en el análisis de sus narrativas, considerando que ellos, como narradores activos, sostienen una estrategia discursiva que disponen conscientemente o no para conseguir sus motivaciones. Esta idea descansa en la concepción de narrativa como "el proceso discursivo constituido por una sucesión de estados y transformaciones que permiten observar la aparición de diferencias y la producción de sentido en un texto a cuyo nivel de superficie pertenece" (Grupo de Entrevernes, 1982, pp. 23-24). Por lo tanto, cuando una narrativa es exhibida socialmente y, en este caso, además se refiere a las noticias como discursos sobre acontecimientos sociales, contribuye a la producción de sentido intersubjetiva y, así, a la construcción de la realidad pública.

En este marco, Twitter ofrece la posibilidad de observar de cerca este fenómeno porque es una red social donde los usuarios tienden a seguir, además de a amigos, a personas con las que tienen intereses en común, medios de comunicación, líderes de opinión y famosos. Mientras que en Facebook, las preferencias se concentran sobre todo en conocidos, es decir, contactos que ya forman parte de las redes sociales del usuario en el mundo analógico (Universidad Católica de Chile \& Gfk Adimark, 2016). En esto influye sin duda el hecho de que Twitter, no así Facebook, permite la existencia de lazos asimétricos, donde un usuario puede seguir una cuenta no protegida sin necesidad de que esta conexión sea recíproca (Golder \& Macy, 2014).

Esto explica que, en nuestros estudios anteriores, hayamos establecido que un tercio de los trending topics en Twitter en Chile correspondan a noticias (Miranda, 2016), lo que lo convierte en un lugar privilegiado para hablar sobre temas públicos.

Esto último se produce aun cuando las redes sociales más utilizadas en Chile sean Whatsapp y Facebook y el uso de Twitter se calcule en un moderado $19 \%$. Sin embargo, está confirmado que hay personas mayores que utilizan esta red social, porque un $10 \%$ de los chilenos con 55 años o más declara ser un tuitero asiduo (Universidad Católica de Chile \& Gfk Adimark, 2016).

\section{Co-constructores de la noticia interactiva}

El uso de las redes sociales digitales en las personas mayores, específicamente en el comentario público de noticias, representa una posibilidad de participación en el proceso de construcción de la realidad social. Esto se base en el concepto de la noticia como un discurso que, además de referir la observación de los acontecimientos del mundo real y nuestros mundos culturales de referencia, está compuesto en forma determinante por el "mundo posible", elaborado por el periodista-narrador. En este fenómeno, los medios tienen un rol privilegiado (Rodrigo, 1989), pero no lo ejercen de forma unívoca porque, lejos de las ideas de manipulación mediática, la interacción con la audiencia es determinante en el consumo de la noticia, en su reconocimiento y, 
en la web 2.0, en la interactividad discursiva que potencialmente produce.

En este contexto, la interactividad es entendida no sólo como el compromiso psicológico necesario para la interpretación de un contenido mediático, sino "como el flujo coherente comunicativo inmersivo multinivel: estético, narrativo y emocional" (Mora-Fernández, 2013, p. 9). De esta manera, un discurso a modo de comentario en una noticia digital requiere un compromiso mental y físico, el despliegue de una narrativa y el compromiso de desnudez emocional que se necesita para sentir que el discurso puede entablar una conexión con el mensaje mediático original, que merece ser exhibido a otros. Tomarse la molestia de generar este texto permite suponer que existe una alta motivación por parte del usuario, tomando en cuenta la regla de oro de la interactividad de Polaine $(2013$, s.p.): "The effort required to experience something should be less than or equal to the payoff of the experience".

En consecuencia, es importante mencionar que, en Estados Unidos, el Engaging News Project (Stroud, Van Duyn, \& Peacock, 2016) concluyó que un 14\% de los usuarios de internet comentan las noticias. De ellos, el 36,9\% mayoritario tiene entre 30 y 49 años. Pero los usuarios de más de 50 años alcanzan un $35,5 \%$, que casi iguala a los adultos jóvenes, lo que sugiere que existe un interés en este grupo por comentar noticias digitalmente, lo que aún no ha sido explorado en Chile.

\section{Metodología propuesta}

La metodología propuesta es cuantitativa, considerando que este estudio pretende identificar los rasgos predominantes de las narrativas de las personas mayores que comentan asuntos de interés públicos en Twitter.

Se realizó un análisis transeccional (Hernández, Fernández-Collado, \& Baptista, 2006) a partir de los perfiles recogidos con la herramienta online Followerwonk, que permite indagar en los perfiles de los tuiteros y que ha sido usada ampliamente en estudios recientes sobre comunicación social en Twitter (An \& Weber, 2016, Barboza et al., 2017, Castillo-Ramírez \& Alberich-Pascual, 2017).

El principal desafío en la recolección de datos fue filtrar a los tuiteros mayores, porque Twitter, a diferencia de Facebook, no pregunta abiertamente la fecha de nacimiento y en general contiene menos datos personales (Golder \& Macy, 2014). Finalmente, se decidió seleccionar aquellos perfiles en cuyas "bios" se usara la palabra "jubilada" o "jubilado", lo que implicó considerar a usuarios que abiertamente confesaran estar cursando la "tercera edad", tomando en cuenta que en Chile la edad mínima de retiro es de 60 años para las mujeres y de 65 para los hombres.

Luego de evaluar la pertinencia de cada uno de los perfiles, se obtuvo una muestra aleatoria de 187 cuentas de adultos mayores chilenos, de un total de 48 ciudades diferentes, considerando que no todos especificaron su lugar de residencia. Un 55\% correspondió a mujeres y un $45 \%$, a hombres, lo que se asimila a la distribución por sexo de la población nacional (INE, 2017).

Posteriormente, se estableció que, de esa muestra de tuiteros mayores, un $32 \%$ presentaba una baja actividad, es decir, tenían menos de 20 publicaciones en total en su cuenta.

A continuación, se recolectaron los últimos 20 tuits de cada usuario de la muestra. Luego, se identificaron los tuits seminales, un término acuñado en nuestros estudios preliminares para determinar a los tuits originales, que no son retuits, ni respuestas a otros usuarios (Fernández y Miranda, 2015).

Además se escogieron los que estaban relacionados con noticias, con temas públicos o con medios de comunicación social.
Esto arrojó un total final de 107 usuarios con 776 tuits seminales de carácter público, es decir, relacionados con noticias, temas sociales o medios de comunicación.

En base a esta muestra, se realizó un análisis de contenido cuantitativo tipo análisis de frecuencia, aplicando indicadores que nos permitieran, en una primera etapa, caracterizar el uso de la plataforma en este segmento etario y, posteriormente, describir las narrativas textuales y digitales predominantes y sus interacciones con otros usuarios.

\section{Resultados}

Se estableció en primer lugar, que más de la mitad los adultos mayores (57\%) tuitearon sobre temas de carácter público. Esto sugiere ya en una primera fase de análisis un interés por la información y la participación en torno a asuntos sociales.

Ésta fue una conducta más frecuente entre los hombres, que tuvieron en promedio un $41 \%$ tuits seminales sobre temas sociales, mientras que las mujeres registraron sólo un 31\%. Esta diferencia podría deberse a antiguos estereotipos etarios que relacionan al hombre con la esfera de lo pLa segunda incidenciae tipo. de los adultos mayores expreso tesis de caracte lo p las mujeres, sson retuits, ni respuestas a otúblico y a la mujer con el espacio doméstico.

Para iniciar el análisis de las narrativas, se aplicaron categorías simples, que mezclan la retórica tradicional aristotélica con la clasificación de titulares de Núñez Ladevéze (como se citó en Zorrilla, 1996). La estrategia más utilizada fue la opinión, que consiste en entregar la "inventio", definida como "la acción de encontrar qué decir" (Marafioti, 2005, p. 45), planteando una tesis de carácter valórico o subjetivo. Un $57 \%$ de los tuits seminales de los adultos mayores expresó una sentencia de este tipo.

\section{Gráfico 1: Uso de narrativas textuales en los tuits seminales de adultos mayores. Fuente: elaboración propia.}

\section{Uso de narrativas textuales en los tuits de adultos mayores}

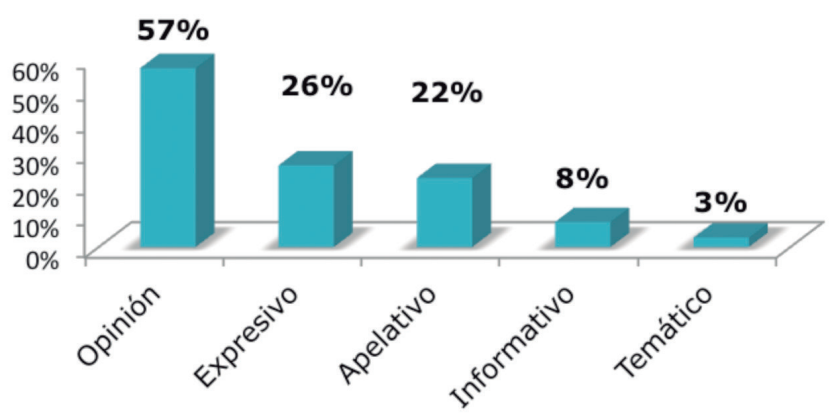

La segunda estrategia narrativa es la expresión, situada en el concepto clásico de elocutio, a través de un sujeto narrador activo, que escoge las palabras y las organiza en la enunciación (Marafioti, 2005). En este caso, se ubicaron dentro de este concepto los modismos expresivos o frases emotivas, que privilegian la forma más allá de lo funcional. Se pudo encontrar esta estrategia en un $26 \%$ de los tuits analizados y correspondió a signos de exclamación, uso de expresiones coloquiales o chilenismos y adjetivos especialmente connotativos.

La tercera incidencia correspondió a los tuits apelativos, que en retórica clásica identificamos como el exordio, es decir, la seducción incial para ganarse la atención y la complicidad del 
usuario (Marafioti, 2005). Este tipo de narrativas estuvo presente en un $22 \%$ de los tuits analizados. Esta actitud discursiva es muy compatible con el concepto digital de interactividad, que consiste en la generación de un bucle de acciones y reacciones entre emisor y destinatario, como diría Murray (1999).

Las narrativas textuales menos utilizadas fueron la informativa, que imita la pirámide invertida, como fórmula tradicional de los medios periodísticos (en un $8 \%$ de los tuits); y la temática (en un 3\%), que sólo enuncia el tópico que se alude.

De todos modos, como se trata de una plataforma digital, no se puede aislar la estrategia discursiva textual sin tomar en cuenta el uso de los recursos multimediales propios de la plataforma, que también constituyen narrativas aunque de tipo digital. Así pudimos observar que un 55\% de los usuarios añadió alguna de estas herramientas a sus enunciados escritos. El resto sólo se conformó con el texto. Probablemente esta conducta se deba a que aún no se ha hecho la transición completa desde la cultura de la imprenta a la de un medio social digital.

Gráfico 2: Uso de recursos digitales en tuits seminales en adultos mayores. Fuente: elaboración propia.

\section{Uso de recursos digitales en tuits seminales de adultos mayores}

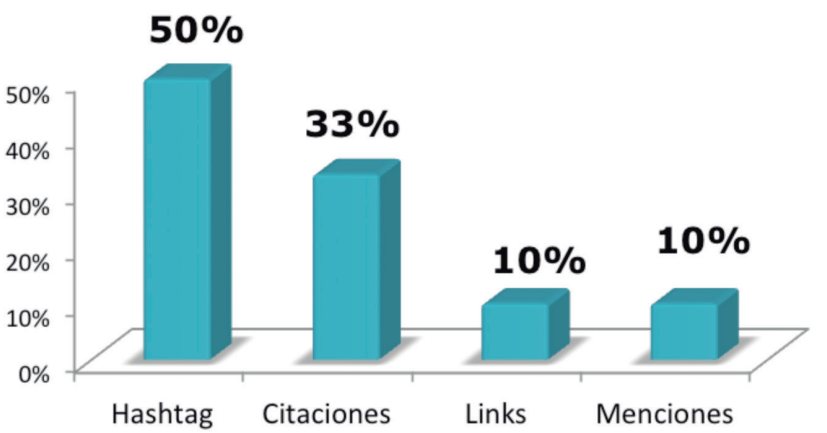

Dentro de los recursos utilizados, el más frecuente fue el hashtag, que estuvo en la mitad de los tuits que aplicaron herramientas digitales. Es de interés este dato porque el hashtag en Twitter funciona como un principio unificador de los temas de conversación. Así, los adultos mayores estarían llamando a la participación a otros usuarios o aunándose voluntariamente a una conversación establecida sobre asuntos de la esfera pública. En la mayoría de los casos (64\%), este hashtag fue acompañado de una opinión, lo que refuerza la idea de la necesidad de opinar sobre un tópico común de carácter público.

El segundo recurso más utilizado ( $33 \%$ de los tuits) fue la citación del tweet de otro usuario, que se incluyó en un tercio de las publicaciones que aplicaron herramientas digitales. El 48\% de ellos se usó también para opinar acerca del planteamiento de esta persona.

En un tercer lugar se ubican las menciones y los links (cada uno aparece en un $10 \%$ de los tuits), que apuntan, respectivamente, a la apelación directa a un usuario definido y a la conexión con un contenido digital, a menudo más detallado que los 140 caracteres que permitía Twitter al momento de la recolección de datos (posteriormente lo amplió a 280 en algunos idiomas (Emol, 2017)).

Otros recursos de mayor complejidad en su producción, como GIFs, memes, videos y fotos, tienen una aparición casi nula en los tuits seminales. Es decir, los tuiteros mayores no suelen compartir recursos de este tipo, de su autoría.
Además, en el uso de estas herramientas, fue posible identificar una tasa de error en el 10\% de los tuits, que involucró al 17\% de los tuiteros mayores: un $12 \%$ de los usuarios hombres y un $22 \%$ de las mujeres. Estos fueron fallos tipográficos en los hashtags o al escribir los nombres de los usuarios mencionados en el tweet, lo que impidió que se lograra contectar estos recursos con los hipervínculos correspondientes.

\section{Síntomas de convergencia}

En el análisis también fue posible identificar factores de convergencia mediática en algunos tuits. Para identificar este rasgo, se tomó el concepto de lenkins (2008), que propone que cada usuario toma distintas versiones de un mismo contenido recogidas desde distintos soportes mediáticos y crea así su propia síntesis en su cabeza. Y, a menudo, usa además nuevamente los medios para compartir este mensaje con otros.

En este sentido, en la muestra, hubo usuarios que usaron hashtags sugeridos por programas de televisión o de radio, al aire en ese momento, o mencionaron las cuentas de estos medios para interactuar con el contenido, haciendo preguntas a los entrevistados o a los presentadores, coincidiendo o rebatiendo los puntos de vista. Esta conducta se vio en un $28 \%$ de los tuits y en un tercio de los tuiteros.

De este modo, un $40 \%$ de las mujeres y un $26 \%$ de los hombres usaron recursos de convergencia mediática. Este importante dato sugiere el surgimiento incipiente de lo que hemos llamado una "conciencia convergente" (Fernández y Miranda, 2015), en este caso en los adultos mayores, que muestra cierta apertura para comenzar a experimentar los medios de comunicación de forma menos secuencial y más fragmentaria, como es propio de los medios digitales.

\section{Esperando respuesta}

Por último, hay mencionar que los resultados muestran también la necesidad de integración de los adultos mayores. Esto debido a que los niveles de interactividad que sus tuits provocaron es mínimo. Sólo un 6\% de los tuits obtuvo respuestas, un 19\% consiguió retweets y un $23 \%$ recolectó me gusta.

Gráfico 3: Índice de seminalidad del tuit. Fuente: Miranda (2016)

Indice de seminalidad del tuit $=\left(\mathrm{RP}^{*} 0,3\right)+\left(\mathrm{RT}^{*} 0,2\right)+\left(\mathrm{MG}^{*} 0,1\right)$

\section{0,6}

Posteriormente, aplicamos el índice de seminalidad de los tuits, un cálculo que hemos propuesto (Miranda, 2016) y que valora las interacciones de otros usuarios con el contenido según el "esfuerzo creativo" (Kaplan \& Haenlein, 2010) de cada una de las posibles respuestas disponibles en la plataforma. De esta manera, toma en cuenta que la respuesta a un tweet es la actitud de mayor involucramiento, porque implica la creación de nuevo contenido a partir del tuit seminal. La segunda es el retuit, porque supone no sólo la adhesión al tuit, sino que el compartirlo casi como propio con los seguidores. El tercero es el me gusta, porque sólo revela una conexión discursiva de tipo aditivo (Renkema, 1999), que señala un simple agrado por el enunciado (Fernández y Miranda, 2015).

Tras aplicar este índice de seminalidad a los tuits de los adultos mayores, se obtuvo un 0,13 , que es un nivel casi nulo de interactividad discursiva. Esto muestra que, pese a sus esfuerzos, 
los usuarios mayores no han conseguido insertarse en la red y entablar las conversaciones que les gustaría estar teniendo.

\section{Conclusiones}

Aunque no es factible por esta vía conocer cuántos adultos mayores están usando Twitter en Chile, al menos pudimos acceder a un grupo de 187 usuarios que representan a distintos puntos geográficos de nuestro país. De ellos sabemos que un tercio tiene una baja actividad en Twitter. También pudimos verificar que más de la mitad (57\%) ha comentado en esta plataforma asuntos de interés público, mayoritariamente hombres.

$\mathrm{Al}$ analizar sus estrategias narrativas, pudimos ver que usan los 140 caracteres sobre todo para enunciar su opinión, también para expresar sus emociones y para llamar la atención de los otros usuarios, lo que es absolutamente coherente con una estrategia discursiva en un medio social digital.

Estos tuiteros hicieron uso también de las herramientas digitales en la construcción de sus discursos. Destaca especialmente el hashtag, que está hecho para llamar a la interacción y representa la intención de unirse a conversaciones sobre el mundo que compartimos.

Dentro de los aspectos positivos también en la integración de los usuarios mayores a Twitter, se detectó que un tercio de estos tuiteros tiene rasgos que apuntan a la convergencia mediática. Esto sugiere que están abiertos a experimentar los medios de una nueva forma, a la manera digital.

En cuanto a los puntos que esbozan desventajas de los adultos mayores en su desempeño en Twitter, se detectaron errores en el uso de estas herramientas en un $17 \%$ de los usuarios. Además, pese a sus esfuerzos discursivos, el nivel de seminalidad de sus tuits es casi inexistente, lo que indica que prácticamente no generan interacción con otros usuarios, precisamente cuando están abordando temas públicos.

Todo esto nos permite afirmar que existe al menos un pequeño grupo de adultos mayores chilenos que son activos tuiteros, que tienen intuitivamente una habilidad para usar las herramientas digitales y que quieren unirse a las conversaciones sobre temas públicos. Sin embargo, falta aún generar instancias de alfabetización digital para estos usuarios mayores deseosos de participar, considerando que la brecha digital es, al menos en Chile, no una barrera de acceso tecnológico, sino que de educación.

Por último, en futuros estudios en esta línea, será necesario complementar los resultados con estudios de los perfiles de los adultos mayores y con información cualitativa sobre la construcción discursiva y entrevistas, para saber de primera fuente qué requieren para formar parte activa de Twitter, como un potencial espacio para construir la realidad social de forma participativa. De esta manera, podremos ayudar a encaminarlos al envejecimiento activo y escuchar mejor lo que tienen para decirnos.

\section{Referencias bibliográficas}

Agudo, S., Pascual, M., y Fombona, J. (2012). Usos de las herramientas digitales entre las personas mayores. Comunicar, 39 (20), 193-201.

Alves, P., \& De Oliveira, R. (2015). Tecnologías de comunicação e interação e envelhecimento humano: a busca da inclusão social pela inclusão digital. Revista Brasileira de Ciências do Envelhecimento Humano, 12(3), 234-244.

An, J., \& Weber, I. (2016). \# greysanatomy vs.\# yankees: Demographics and Hashtag Use on Twitter. Proceedings of the Tenth International AAAI Conference on Web and Social Media, 523526.
Barboza, M., Blanco, L., Meleán, R., Páez, A., Silva, P., y Villasmil, M. (2017). Una lectura cibergráfica sobre la interactividad en las cuentas de Twitter que manejan los periodistas venezolanos. Temas de Comunicación 33, 162-188.

Bourdieu, P. (1980). Le capital social. Actes de la recherche en sciences sociales, 31, 2-3.

Castillo-Ramírez, I., y Alberich-Pascual, J. (2017). Análisis de estrategias de difusión de contenidos y actividad en redes sociales en revistas de divulgación científica: factores de interacción, visibilidad e impacto. Estudios sobre el Mensaje Periodístico, 23(2), 1045-1056.

Chakraborty, R., Vishik, C., \& Rao, H. (2013). Privacy preserving actions of older adults on social media: Exploring the behavior of opting out of information sharing. Decision Support Systems , 4(55), 948-956.

Chou, W., Lai, Y., \& Liu, K. (2013). User requirements of social media for the elderly: a case study in Taiwan. Behaviour $\mathcal{E}$ Information Technology , 32(9), 920-937.

Ciboh, R. (2017). An Exploratory Study of Older Adults Social Media Use and Social Capital in Nigeria. Athens Journal of Mass Media and Communications, 3(2), 149-165.

Condeza, R., Bastías, G., Valdivia, G., Cheix, C., Barrios, X., Rojas, R., Gálvez, M. y Fernández, F. (2016). Adultos mayores en Chile: descripción de sus necesidades en comunicación en salud preventiva. Cuadernos. info, 28, 85-104.

Dos Santos, R., \& Gasparetto, E. (2016). Literacy in the digital computer education for elderly. Revista Científica Intellectus, 4(35), 53-66.

El-Sahili, L. (2014). Psicología de Facebook: Vislumbrando los fenómenos psíquicos, complejidad y alcance de la red social más grande del mundo. Guanajuato, México: Pontificia Universidad de Guanajuato.

Ellison, N. B. (2007). The benefits of Facebook "friends:" Social capital and college students' use of online social network sites. Journal of Computer-Mediated Communication, 12(4), 11431168.

Emol (2017). Twitter comienza una prueba que permite escribir hasta 280 caracteres en sus publicaciones Recuperado de: http://www. emol.com/noticias/Tecnologia/2017/09/26/876803/Twitter-comienza-una-prueba-que-permitira-escribir-hasta-280-caracteres-en-sus-publicaciones.html

Farias, J., Vitor, T., Lins, P., Pedroza, A., \& Eduardo, L. (2015). Digital inclusion of elderly: a study on the propensity to adoption of information and communication technologies (ICTs). Revista Gestão \& Tecnologia , 15(3), 164-188.

Fernández, F., y Miranda, M. (2015). Hablándole a la televisión: análisis de las conexiones discursivas entre Twitter y tres programas de contenido político en televisión abierta. Comunicación y Sociedad, 24, 71-94.

Fernández, M., y Herrera, M. (2016). Chile y sus mayores. 10 años de la Encuesta Calidad de Vida en la Vejez UC - Caja Los Andes. Recuperado de: http://www.senama.cl/filesapp/Chile\%20 y\%20sus\%20mayores\%202013,\%20Encuesta\%20de\%20Calidad\%20de\%20Vida.pdf

Girlado, L., Leal, S., \& Lilia, L. (2015). Las posibilidades de internet en la reconfiguración de la red social primaria del adulto mayor, fracturada por efecto de la migración. El caso de los adultos mayores con parientes migrantes en 6 municipios del departamento del Quindío. Anagramas, 14(27), 145-162.

Godoy, S., y Gálvez, M. (2015). Una mirada al futuro probable: análisis de escenario para implementar en 2020 un sistema de comunicación y educación en salud para adultos mayores. En INCOM, Asociación Chilena de Investigadores en Comunicación. La comunicación y sus desafíos para la investigación e 
investigadores del siglo XXI: perspectivas en contextos de incertidumbre (pp. 76-86). Antofagasta, Chile: INCOM.

Golder, S., \& Macy, M. (2014). Digital Footprints: Opportunities and Challenges for Online Social Research. Annual Review of Sociology, 40, 129-152.

Grupo de Entrevernes (1982). Análisis semiótico de los textos: introducción, teoría, práctica. Madrid: Cristiandad.

Hernández, R., Fernández-Collado, C., y Baptista, P. (2006). Metodología de la Investigación. México, México: Mc Graw Hill Interamericana.

INE (2017). Resultados definitivos CENSO 2017. Recuperado de: http://www.censo2017.cl/wp-content/uploads/2017/12/Presentacion_Resultados_Definitivos_Censo2017.pdf

INE (2012). Demográficas y vitales. Recuperado de: http://www. ine.cl/canales/chile_estadistico/familias/demograficas_vitales.php

INE (2015). Encuesta suplementaria de ingresos. Recuperado de: http://www.ine.cl/canales/chile_estadistico/mercado_del_ trabajo/nene/esi/index.php

Jenkins, H. (2008). Convergence culture. La cultura de la convergencia de los medios de comunicación. Barcelona: Paidós.

Jung, E., Walden, J., Celeste, A., \& Shyam, S. (2017). Social Networking in the Aging Context: Why Older Adults Use or Avoid Facebook. Telematics and Informatics, 34(7), 1-10.

Kaplan, A., \& Haenlein, M. (2010). Users of the world, unite! The challenges and opportunities of Social Media. Business horizons, 53(1), 59-68.

Llorente-Barroso, C., Iñarás-Abad, M., \& Sánchez-Valle, M. (2015). Mayores e Internet: La Red como fuente de oportunidades para un envejecimiento activo. Comunicar: Revista Científica de Comunicación y Educación, 23(45), 29-36.

Marafioti, R. (2005). Los patrones de la argumentación. La argumentación en los clásicos y en el siglo XX. Buenos Aires, Argentina: Biblos.

Miranda, M. (2016). ¿Dónde está el narrador? Las narrativas de la noticia en Twitter. El tiempo, el espacio y el sujeto en 140 caracteres. Tesis doctoral. Universidad Nacional de Cuyo. Mendoza, Argentina.

Mora-Fernández, J. (2013). Artecnología en cine interactivo: algunas categorías, interfaces, estructuras narrativas, emociones e investigaciones. En Andrade, V., \& A. Colorado, A. (ed.), ArTecnologia: arte, tecnologia e linguagens midiáticas. Porto Alegre, Brasil: Buqui.

Morgan, D. (2014). Tweet Up? Examining Twitter's Impact on Social Capital and Digital Citizenship in Higher Education. About campus, 18(6), 10-17.

Murray, J. (1999). Hamlet en la holocubierta. El futuro de la narrativa en el ciberespacio. Barcelona: Paidós.

Nazar, G., \& Figueroa, C. (2015). Creencias estereotípicas sobre el desempeño laboral de trabajadores mayores en Chile. Psicoperspectivas. Individuo y sociedad, 14(1), 114-125.

Niehaves, B., \& Plattfaut, R. (2014). Internet adoption by the elderly: employing IS technology acceptance theories for understanding the age-related digital divide. European Journal of Information Systems: An Official Journal of the Operational Research Society, 23(6), 708-726.
Palma-Candia, O., Hueso-Montoro, C., Ortega-Valdivieso, A., Montoya-Juárez, R., y Cruz-Quintana, F. (2016). Factores sociodemográficos y de participación social relacionados con el bienestar psicológico en adultos mayores en la región de Magallanes, Chile. Revista Médica de Chile, 144(10), 1287-1296.

Peral-Peral, B., Arenas-Gaitán, J., \& Villarejo-Ramos, A. (2015). From digital divide to psycho-digital divide: Elders and online social networks. Comunicar, 23(45), 57-64.

Polaine, A. (28 de febrero de 2013). A Golden Rule for Interactions and Life. [Mensaje en un blog]. Recuperado de http:// www.polaine.com/tag/interactivity/

Ramón-Jerónimo, M., Peral-Peral, B., \& Arenas-Gaitán, J. (2013). Elderly Persons and Internet Use. Social Science Computer Review, 31(4), 389-403.

Rasi, P., \& Kilpeläiner, A. (2015). The digital competences and agency of older people living in rural villages in finnish Lapland. Media, Technology E Life-Long Learning, 11(2), 149160.

Renkema, J. (1999). Introducción a los estudios sobre el discurso. Barcelona: Gedisa.

Richardson, J. E. (2008). Readers' letters. En Franklin, B. Pulling newspapers apart: Analysing print journalism (pp. 56-66). Nueva York, Estados Unidos: Routledge.

Rodrigo, M. (1989). La construcción social de la noticia. Barcelona, España: Paidós.

Rogers, E. (1983). Diffusion of innovations. New York, Estados Unidos: The Free Press.

Rojas, M., Carvajal, M., y Kornfeld, R. (2011). Participación social, un derecho para un mejor envejecer. En C. Bru (2011), IV Congreso Iberoamericano de Universidades para Adultos Mayores CIUUMM 2011 (pp. 895-899). Alicante, España: AEPUM.

Sæbø, Ø., Resca , A., \& Spagnoletti, P. (2015). Reframing health care through social media. Journal of Information Technology Teaching Cases, 5(2), 65-73.

Sergeyeva, O., \& Makarova, L. (2016). E-inclusion and perception of time among elderly: Russian regional perspective. International Journal of Electronic Governance, 8(3), 303-313.

Stroud, N., Van Duyn, E., \& Peacock, C. (2016). News commenters and news comment readers. Recuperado de: https://engagingnewsproject.org/wp-content/uploads/2016/03/ENP-NewsCommenters-and-Comment-Readers1.pdf

Tsai, T., Chang, H., \& Ho, Y. (2016). Perceptions of a Specific Family Communication Application among Grandparents and Grandchildren: An Extension of the Technology Acceptance Model. Plos One, 11(6), 1-23.

Universidad Católica de Chile \& Gfk Adimark. (2016). Encuesta Bicentenario. Recuperado de: http://politicaspublicas.uc.cl/ wp-content/uploads/2017/01/Encuesta-bicentenario-2016. pdf

WHO (2002). Active ageing. A policy framework. Recuperado de: http://apps.who.int/iris/bitstream/10665/67215/1/WHO_ NMH_NPH_02.8.pdf

Young, K. (2011). Social ties, social networks and the Facebook experience. International Journal of Emerging Technologies and Society, 1(9), 20-34.

Zorrilla, J. (1996). El titular de la noticia. Estudio de los titulares informativos en los diarios de difusión nacional. Tesis doctoral. Universidad Complutense de Madrid. Madrid, España. 
